\title{
Momentum space dipole amplitude for DIS and inclusive hadron production
}

\section{B. Gay Ducati*}

Instituto de Física, Universidade Federal do Rio Grande do Sul, Caixa Postal 15051, 91501-970, Porto Alegre, RS, Brazil

E-mail: beatriz.gay@ufrgs.br

\section{E. A. F. Basso}

Instituto de Física, Universidade Federal do Rio Grande do Sul, Caixa Postal 15051, 91501-970, Porto Alegre, RS, Brazil

E-mail: andre.basso@ufrgs.br

\section{E. G. de Oliveira}

Instituto de Física, Universidade de São Paulo, Caixa Postal 66318, 05314-970, São Paulo, SP,

Brazil

E-mail: egdeoliveira@if.usp.br

We present a global analysis for DIS and inclusive hadron production data of a dipole model entirely constructed in momentum space. The inclusive hadron yield was described within a hybrid factorization formalism for the production cross section that considers the projectile through a DGLAP evolved parton distribution function, while the target is seen as a dense partonic system. The results of the fit show good agreement with the first CMS data for proton-proton collisions at LHC, in spite of the large $K$ factors. With the use of the $k_{t}$ factorization formalism, it is shown that such errors are mainly due to uncertainties in the hybrid formalism when dealing with central rapidity data.

PACS: $12.38 . \mathrm{Bx}, 13.60 . \mathrm{Hb}, 13.85 . \mathrm{Ni}$

36th International Conference on High Energy Physics,

July 4-11, 2012

Melbourne, Australia

\footnotetext{
* Speaker.
} 


\section{Small- $x$ evolution and the AGBS dipole model}

At high energies, when the momentum fraction of the partons inside the hadron becomes very small, the QCD evolution in $\log (x)$ becomes nonlinear in order to tame the fast growing of partons, created by Bremsstrahlung as the energy increases. In this region there is a particular frame, the dipole frame, where the deep inelastic scattering (DIS) process can be factorized as follows: the virtual photon probing the target can split into a quark-antiquark pair, or dipole, though the proton still carries most of the total energy. The cross section can be written as

$$
\sigma_{T, L}^{\gamma^{*} p}\left(Q^{2}, Y\right)=\int d^{2} r \int_{0}^{1} d z\left|\Psi_{T, L}\left(\boldsymbol{r}, z ; Q^{2}\right)\right|^{2} \sigma_{\mathrm{dip}}(\boldsymbol{r}, Y)
$$

where the labels $T$ and $L$ refer, respectively, to the transverse and longitudinal parts of the cross section, $\boldsymbol{x}$ and $\boldsymbol{y}$ are the transverse coordinates of the quark and the antiquark, being $\boldsymbol{r}=\boldsymbol{x}-\boldsymbol{y}$ the transverse size of the dipole; $z$ is the momentum fraction of the photon carried by the quark and $\Psi_{T, L}\left(\boldsymbol{r}, z ; Q^{2}\right)$ are the transverse and longitudinal wavefunctions for the photon in the dipole description. The quantity $\sigma_{\text {dip }}$ in Eq.(1.1) is the dipole-proton cross section which can be expressed as

$$
\sigma_{\text {dip }}^{\gamma^{*} p}(r, Y)=2 \pi R_{p}^{2} \mathscr{N}(r, Y) .
$$

where $\mathscr{N}(r, Y)$ is the dipole scattering amplitude in the coordinate space.

The non linear evolution of such amplitude can be describe in a simple way through the socalled Balitsky-Kovchegov equation [1], which can be written in momentum space as

$$
\partial_{Y} N(k, Y)=\bar{\alpha} \chi\left(-\partial_{L}\right) N(k, Y)-\bar{\alpha} N(k, Y)^{2},
$$

where $\chi(\gamma)=2 \psi(1)-\psi(\gamma)-\psi(1-\gamma)$ is the BFKL [2] kernel and $L=\log \left(k^{2} / k_{0}^{2}\right)$, being $k_{0}$ a fixed soft scale. This equation gives the rapidity evolution of the color dipole with transverse momentum $k \sim 1 / r$, being the non linear term responsible for the unitarization of the amplitude in the low $k$ (large $r$ ) limit. A parametrization of dipole-proton scattering amplitude in momentum space has been proposed by de Santana Amaral, Gay Ducati, Betemps, and Soyez (AGBS) [3]. It interpolates analytically between the behavior of the Balitsky-Kovchegov (BK) [1] evolution equation solutions in the dilute regime

$$
N(k, Y) \stackrel{k \gg Q_{s}}{\approx}\left(\frac{k^{2}}{Q_{s}^{2}(Y)}\right)^{-\gamma_{c}} \log \left(\frac{k^{2}}{Q_{s}^{2}(Y)}\right) \exp \left[-\frac{\log ^{2}\left(k^{2} / Q_{s}^{2}(Y)\right)}{2 \bar{\alpha} \chi^{\prime \prime}\left(\gamma_{c}\right) Y}\right],
$$

and the saturation one, in which it behaves like

$$
N(k, Y) \stackrel{k \ll Q_{s}}{=} c-\log \left(\frac{k}{Q_{s}(Y)}\right) .
$$

The interpolation in the AGBS model is done through the following expression for the scattering amplitude $\left(\rho \equiv \ln \left(k^{2} / k_{0}^{2}\right)\right.$ and $\left.\rho_{s} \equiv \ln \left(k_{0}^{2} / Q_{s}^{2}\right)\right)$

$$
\tilde{T}^{\mathrm{AGBS}}(\rho, Y)=L_{F}\left(1-e^{-T_{\mathrm{dil}}}\right), \quad \text { where } \quad L_{F}=1+\ln \left[e^{\frac{1}{2}\left(\rho-\rho_{s}\right)}+e^{-\frac{1}{2}\left(\rho-\rho_{s}\right)}\right],
$$

and

$$
T_{\mathrm{dil}}=\exp \left[-\gamma_{c}\left(\rho-\rho_{s}\right)-\frac{\ln \left[1+e^{\left(\rho-\rho_{s}\right)}\right]^{2}-\log ^{2}(2)}{2 \bar{\alpha} \chi^{\prime \prime}\left(\gamma_{c}\right) Y}\right],
$$


being the saturation scale defined as $Q_{s}^{2}(Y)=k_{0}^{2} e^{\lambda Y}$. This model describes quite well the HERA data on $F_{2}^{p}$ with heavy quarks included [3] and was also used to study whether the possible effects of pomeron loops on the high energy QCD evolution were already present at HERA energies [4].

\section{Global fit to HERA and RHIC data}

Recently the AGBS model was globally fitted to DIS and inclusive hadron production in proton-proton and proton nucleus collisions. The HERA proton structure function $F_{2}^{p}$ is written as

$$
F_{2}\left(x, Q^{2}\right)=\frac{Q^{2} R_{p}^{2} N_{c}}{4 \pi^{2}} \int_{0}^{\infty} \frac{d k}{k} \int_{0}^{1} d z\left|\tilde{\Psi}\left(k^{2}, z ; Q^{2}\right)\right|^{2} N(k, Y)
$$

where now the photon wavefunction is expressed in momentum space and $N(k, Y)$ is the scattering amplitude in momentum space. The RHIC inclusive hadron yield assumes a hybrid factorization

$$
\begin{aligned}
\frac{d N}{d y_{h} d^{2} p_{t}}=\frac{K}{(2 \pi)^{2}} \int_{x_{F}}^{1} \frac{d z}{z}[ & x_{1} f_{q / p}\left(x_{1}, p_{t}^{2}\right) \tilde{N}_{F}\left(\frac{p_{t}}{z}, x_{2}\right) D_{h / q}\left(z, p_{t}^{2}\right) \\
& \left.+x_{1} f_{g / p}\left(x_{1}, p_{t}^{2}\right) \tilde{N}_{A}\left(\frac{p_{t}}{z}, x_{2}\right) D_{h / g}\left(z, p_{t}^{2}\right)\right],
\end{aligned}
$$

where $p_{t}$ and $y_{h}$ are the transverse momentum and rapidity of the produced hadron while $f_{i / p}$ and $D_{h / i}$ refer to the parton distribution function (PDF) of the incoming nucleon and to the hadron fragmentation function (FF) respectively, which are considered at the scale $Q^{2}=p_{t}^{2}>1 \mathrm{GeV}^{2}$. Here one used the CTEQ6 LO PDFs [8] and the LO KKP FF [9]. $N_{A, F}$ denote the scattering amplitudes in the adjoint and fundamental representation, respectively. The data set used in the fit was defined within the following kinematical range: The $F_{2}^{p}$ from the last combined HERA data [6] was taken for $x \leq 0.01$ and $0.1 \leq Q^{2} \leq 150 \mathrm{GeV}^{2}$; The inclusive charged hadron production data from RHIC [7] was considered for $p_{t} \geq 1 \mathrm{GeV}$ and in two rapidity regions - the forward one $\left(y_{h} \geq 2\right)$ and at midrapidities $\left(y_{h} \geq 1\right)$. We set $\bar{\alpha}=0.2$ and $\gamma_{c}=0.6275$, while the other parameters in the amplitude $-\lambda, k_{0}^{2}$ and $\chi^{\prime \prime}\left(\gamma_{c}\right)$ - are left to be free. The normalization factors were also allowed to vary: the proton radius $R_{p}$ and the rapidity-dependent $K$ factors.

\begin{tabular}{|c|c|c|c|c|}
\hline$\chi^{2} /$ d.o.f. & $k_{0}^{2}\left(\times 10^{-3}\right)$ & $\lambda$ & $\chi^{\prime \prime}\left(\gamma_{c}\right)$ & $R\left(\mathrm{GeV}^{-1}\right)$ \\
\hline \hline 0.903 & $1.13 \pm 0.024$ & $0.165 \pm 0.002$ & $7.488 \pm 0.081$ & $5.490 \pm 0.039$ \\
\hline
\end{tabular}

Table 1: Parameters extracted from the fit to H1 and ZEUS combined data [6] on the proton structure function $F_{2}$ at HERA.

Before proceed with the global data analysis we performed a fit to the new HERA data, in order to verify how the model would behave against the new combined H1 and ZEUS data [6]. The parameters, shown in the table 1 did not changed significantly in relation to the original AGBS fit, and this fit is our guideline for the global one, once DIS processes are free from the uncertainties embedded in the PDFs and FFs. The results of the fit are shown in the Fig. 1. One can see by the $\chi^{2}$ values of the table 2 that the fit is better when only forward RHIC data were considered. This is so because the in mid rapidity region the target has not reached its gluon condensate state, when neither our amplitude nor the CGC formulation entering the Eq. (2.2) are valid. All in all, the parameters show that the AGBS model describes equally well the HERA and RHIC data, and the last improves the AGBS model. 

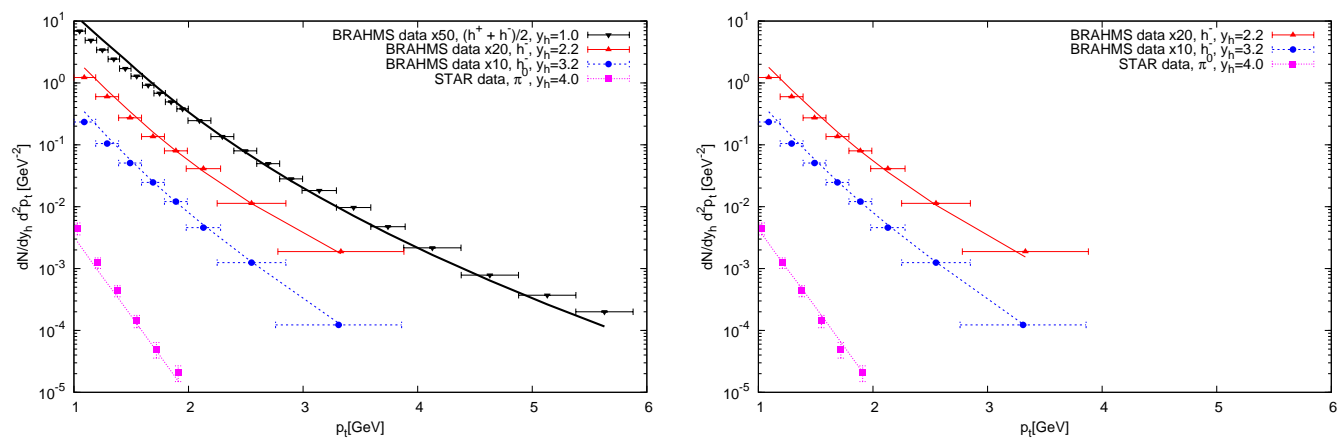

Figure 1: Results for the RHIC charged hadron and $\pi^{0}$ yield for $d+A u$ collisions from the simultaneous fit of AGBS to RHIC [7] and HERA [6] data. Left plot includes the midrapidity region $y_{h} \geq 1.0$.

\begin{tabular}{|c||c|c|}
\hline & $y_{h} \geq 2.2$ & $y_{h} \geq 1.0$ \\
\hline \hline$\chi^{2} /$ d.o.f. & 0.799 & 1.056 \\
\hline$k_{0}^{2}\left(\times 10^{-3}\right)$ & $2.760 \pm 0.130$ & $1.660 \pm 0.137$ \\
\hline$\lambda$ & $0.190 \pm 0.003$ & $0.186 \pm 0.003$ \\
\hline$\chi^{\prime \prime}\left(\gamma_{c}\right)$ & $5.285 \pm 0.123$ & $6.698 \pm 0.223$ \\
\hline$R\left(\mathrm{GeV}^{-1}\right)$ & $4.174 \pm 0.053$ & $4.695 \pm 0.112$ \\
\hline$K\left(y_{h}=1.0\right)$ & - & $6.172 \pm 0.379$ \\
\hline$K\left(y_{h}=2.2\right)$ & $2.816 \pm 0.110$ & $3.783 \pm 0.259$ \\
\hline$K\left(y_{h}=3.2\right)$ & $2.390 \pm 0.098$ & $3.256 \pm 0.226$ \\
\hline$K\left(y_{h}=4.0\right)$ & 0.7 & 0.7 \\
\hline
\end{tabular}

Table 2: Parameters extracted from the global fit to HERA $F_{2}$ data [6] and to the RHIC hadron yield for the $d+A u$ collisions data [7].

\subsection{LHC predictions}

We applied the resulting fit to the forward rapidity region (first line of the table 2) to predict the behavior of LHC hadron yield for $p+p$ and $p+P b$ collisions at energies of 14 and $8.8 \mathrm{TeV}$, respectively. We also confront our calculation with the first data on single inclusive hadron production for $p+p$ collisions measured by the CMS collaboration at the LHC [10]. The results depicted in Fig. 2 show a very good agreement with data, though the $K$ factors are large. This could be explained as an uncertainty of the AGBS model, as well as of the hybrid formalism, in the comparison with the pseudorapidity averaged data performed by the CMS collaboration. Both the AGBS model and the hybrid formalism we used here are designed to study the forward regions, where the target (projectile) wavefunction has a very small- $x$. The data, however, is averaged over the region $|\eta|<2.4$, which imply some disagreements absorbed in the $K$ factors.

\section{$2.2 k_{t}$-factorization formalism}

We also used the $k_{t}$-factorized formalism of [11] to describe the LHC data. In such formalism both colliding partons can carry small- $x$ values, being well suited to study the central rapidity region of produced particles. The cross section is written as

$$
\frac{d \sigma^{A+B \rightarrow g}}{d y d^{2} p_{t}}=K \frac{2}{C_{F} p_{t}^{2}} \int^{p_{t}} \frac{d^{2} k_{t}}{4} \alpha_{s}(Q) \varphi\left(\frac{\left|p_{t}+k_{t}\right|}{2}, x_{1}\right) \varphi\left(\frac{\left|p_{t}-k_{t}\right|}{2}, x_{2} ;\right),
$$




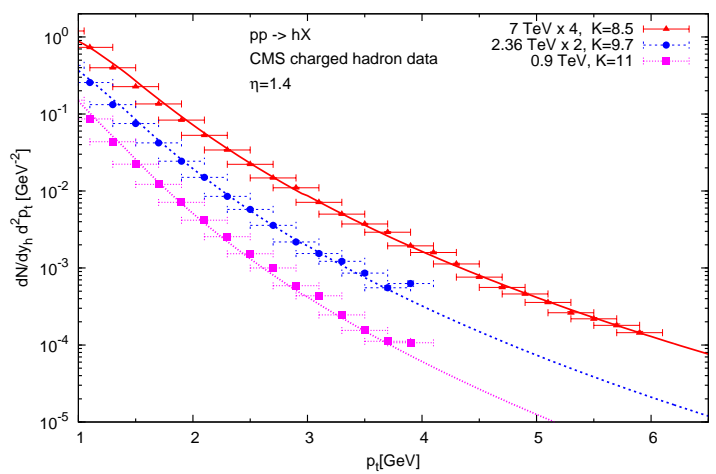

Figure 2: Predictions of the AGBS model to the LHC CMS charged hadron yield for $p+p$ collisions at $\sqrt{s}=0.9,2.36$ and $7 \mathrm{TeV}$. The experimental points are from CMS for $|\eta|<2.4[10]$.
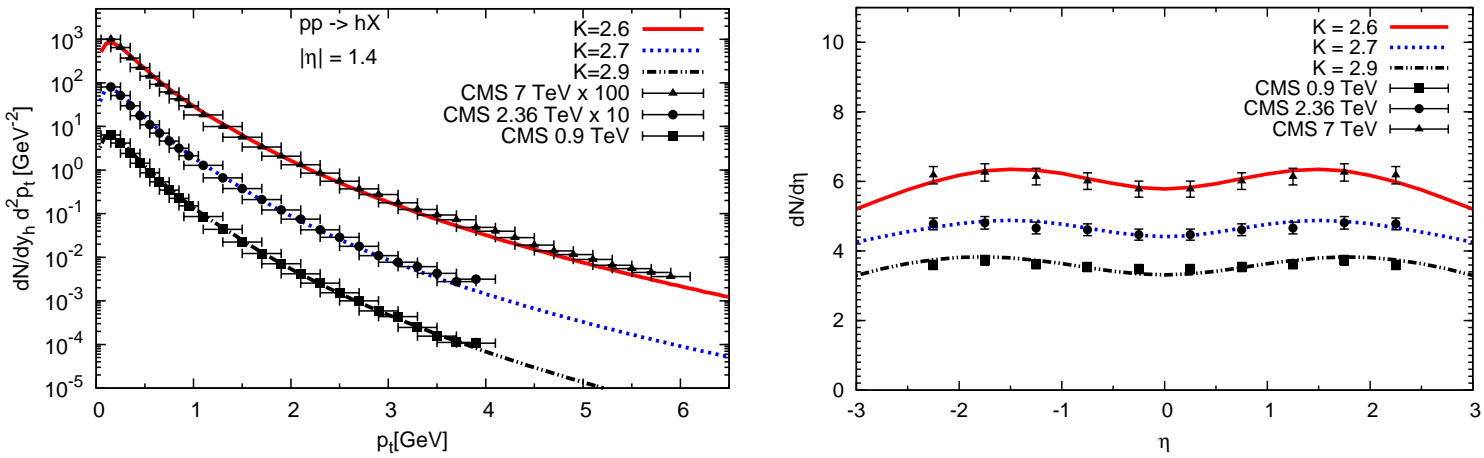

Figure 3: Predictions of the AGBS model to the LHC CMS charged hadron yield for $p+p$ collisions at $\sqrt{s}=0.9,2.36$ and $7 \mathrm{TeV}[10]$.

where $x_{1,2}=\left(p_{t} / \sqrt{s}\right) e^{ \pm y}$ are momentum fractions of the incoming gluons and $C_{F}=\left(N_{c}^{2}-1\right) / 2 N_{c}$ is the Casimir for the fundamental representation. The charged hadron yield, given in terms of (2.3), reads

$$
\frac{d N_{c h}}{d \eta d^{2} p_{t}}=\frac{h[\eta]}{\sigma_{n s d}} \int \frac{d z}{z^{2}} D_{h}\left(z=p_{t} / k_{t}, \mu\right) \frac{d \sigma^{A+B \rightarrow g}}{d y d^{2} p_{t}}
$$

where $D_{h}\left(z=p_{t} / k_{t}, \mu\right)$ stands for the fragmentation function of the produced gluon into hadrons, for which we used the LO KKP model [9] with the scale $\mu=p_{t}$ of the hadron, and $\sigma_{n s d}$ is the non-single-diffractive cross section taken from the KMR model [12]. In the large $N_{c}$ limit, the unintegrated gluon distribution in either of the two colliding hadrons can be related to the dipole scattering amplitude through

$$
\varphi(k, x ; b)=\frac{N_{c}}{2 \pi^{2} \alpha_{s}(k)} k^{2} \nabla_{k}^{2} N_{G}(k, x ; b) .
$$

where $N_{G}$ is the AGBS dipole amplitude in the adjoint representation. The model describes quite well the LHC data [10] on both the high and small $p_{t}$ regions, as seen in Figure 3, and the error associated with the central rapidity data in the hybrid formalism is smaller in this case. 


\section{Discussion}

We revised the global analysis done with the AGBS against the HERA DIS and the RHIC inclusive hadron production data. The calculation used a hybrid formalism for the inclusive cross section, where the projectiles are treated as DGLAP evolved PDFs while the targets considered as a color glass condensate. The fit was better when only the forward RHIC data was considered, meaning that neither the dipole model nor the hybrid formalism are suited to deal with central rapidity data. This becomes more clear when we applied the fit to describe the CMS data on the hadron yield for $p+p$ collisions [10] at different energies. The large $K$ factors include the errors associated with the averaged data over $|\eta|<2.4$ delivered by the CMS collaboration. We also tested the model within the $k_{t}$-factorization formalism, for which the description of the LHC data was quite good. The $K$ factors smaller compared to the hybrid formalism, corroborating the idea the large $K$ gotten in the hybrid formalism were due to the a misleading use of such formalism in a kinematical region it is not designed to work.

\section{Acknowledgments}

This work is supported by CNPq (Brazil). E.G.O. is supported by FAPESP under contract 2011/50597-8.

\section{References}

[1] I. Balitsky, Nucl. Phys. B 463, 99 (1996); Y. V. Kovchegov, Phys. Rev. D 60, 034008 (1999); Phys. Rev. D 61, 074018 (2000).

[2] L. N. Lipatov, Sov. J. Nucl. Phys. 23, 338 (1976); E. A. Kuraev, L. N. Lipatov and V. S. Fadin, Sov. Phys. JETP 45, 199 (1977); I. I. Balitsky and L. N. Lipatov, Sov. J. Nucl. Phys. 28, 822 (1978).

[3] J. T. de Santana Amaral, M. B. Gay Ducati, M. A. Betemps and G. Soyez, Phys. Rev. D 76, 094018 (2007)

[4] E. Basso, M. B. Gay Ducati, E. G. de Oliveira, and J. T. de Santana Amaral, Eur. Phys. J. C 58, 9 (2008); E. Basso, M. B. Gay Ducati, E. G. de Oliveira, and J. T. de Santana Amaral, Braz. J. Phys. v. 38, 483 (2008).

[5] E. A. F. Basso, M. B. Gay Ducati and E. G. de Oliveira, Phys. Rev. D 84, 034024 (2011).

[6] F. D. Aaron et al. (H1 and ZEUS Collaborations), JHEP 0110109 (2010).

[7] I. Arsene et al. (BRAHMS Collaboration), Phys. Rev. Lett. 93, 242303 (2004); J. Adams et al. (STAR collaboration), Phys. Rev. Lett. 97, 152302 (2006).

[8] J. Pumplin et al., JHEP 0712 (2002).

[9] B. A. Kniehl, G. Krammer and B. Pötter, Nucl. Phys. B582, 514 (2000).

[10] V. Khachatryan et al. (CMS Collaboration), JHEP 0210, 041 (2010) [arXiv:hep-ex/1002.0621]; Phys. Rev. Lett. 105, 022002 (2010).

[11] M. Braun, Phys. Lett. B 483, 105 (2000); Y. V. Kovchegov and K. Tuchin, Phys. Rev. D 65, 074026 (2002).

[12] M. G. Ryskin, A. D. Martin and V. A. Khoze, Eur. Phys. J. C 71 (2011) 1617. 